\title{
Mathematical Programming Approach to the Optimality of the Solution for Deterministic Inventory Models with Partial Backordering
}

\author{
Irena Stojkovska \\ Department of Mathematics, Faculty of Natural Sciences and Mathematics, Saints Cyril and Methodius University, Gazi Baba bb, \\ 1000 Skopje, Macedonia
}

Correspondence should be addressed to Irena Stojkovska; irena.stojkovska@gmail.com

Received 26 March 2013; Accepted 5 June 2013

Academic Editor: Michael N. Katehakis

Copyright (C) 2013 Irena Stojkovska. This is an open access article distributed under the Creative Commons Attribution License, which permits unrestricted use, distribution, and reproduction in any medium, provided the original work is properly cited.

We give an alternative proof of the optimality of the solution for the deterministic EPQ with partial backordering (EPQ-PBO) [Omega, vol. 37, no. 3, pp. 624-636, 2009]. Our proof is based on the mathematical programming theory. We also demonstrate the determination of the optimal decision policy through solving the corresponding mathematical programming problem. We indicate that the same approach can be used within other inventory models with partial backordering, and we consider additional models.

\section{Introduction}

A basic inventory control model is the economic order quantity (EOQ) model known by its simplicity and its restrictive modeling assumptions. Managing the inventory of a single item the purpose of the EOQ model is to determine how much to purchase (order quantity) and when to place the order (the reorder point). By relaxing the assumption that shortages are not allowed and allowing stockouts with partial backordering, the EOQ will extend to EOQ with partial backordering (EOQ-PBO). But when the assumption of instantaneous replenishment is replaced with the assumption that the replenishment order is received at a constant finite rate over time, EOQ is extended to the economic production quantity (EPQ) model, and by allowing stockouts with partial backordering it will extend to EPQ with partial backordering (EPQ$\mathrm{PBO})$. There are many papers that propose an inventory model with partial backordering; a very few of them are [1-7]. For a survey of the deterministic models for the EOQ and EPQ with partial backordering, see Penticio's and Drake's paper [8].

Pentico et al. [5] proposed EPQ-PBO model, whose main characteristics are that the derived equations are more like those for the classical EPQ model and it gives the optimal solution in a closed form that helps in understanding the behavior of the inventory system. Let us briefly give the notations from [5] that are used in the total cost function and in the solution of the Pentico et al's EPQ-PBO as well. They are

$D$ : demand per year,

$P$ : production rate per year if constantly producing,

$C_{0}$ : the fixed cost of placing and receiving an order,

$C_{h}$ : the cost to hold a unit in inventory for a year,

$C_{b}$ : the cost to keep a unit backordered for a year,

$C_{1}$ : the cost for a lost sale,

$\beta$ : the backordering rate,

$T$ : the length of an order cycle (a decision variable),

$F$ : the fill rate (a decision variable).

The derived total averaged cost per year for the Pentico et al.'s EPQ-PBO model, according to [5], that has to be minimized is

$$
\Gamma(T, F)=\frac{C_{0}}{T}+\frac{C_{h}^{\prime} D T F^{2}}{2}+\frac{\beta C_{b}^{\prime} D T(1-F)^{2}}{2}+C_{1}^{\prime} D(1-F),
$$


where $C_{h}^{\prime}=C_{h}(1-D / P), C_{b}^{\prime}=C_{b}(1-\beta D / P)$, and $C_{1}^{\prime}=$ $C_{1}(1-\beta)$. Setting the first partial derivatives of $\Gamma(T, F)$ equal to zero, Pentico et al. [5] find the following solutions:

$$
\begin{gathered}
T^{*}=\sqrt{\frac{2 C_{0}}{D C_{h}^{\prime}}\left(\frac{C_{h}^{\prime}+\beta C_{b}^{\prime}}{\beta C_{b}^{\prime}}\right)-\frac{\left(C_{1}^{\prime}\right)^{2}}{\beta C_{h}^{\prime} C_{b}^{\prime}}} \\
F^{*}=F\left(T^{*}\right)=\frac{C_{1}^{\prime}+\beta C_{b}^{\prime} T^{*}}{T^{*}\left(C_{h}^{\prime}+\beta C_{b}^{\prime}\right)} .
\end{gathered}
$$

Comparing the optimal time length of inventory cycle $T^{*}$ for the partial backordering model with the one for the standard EPQ model if backordering is optimal, Pentico et al. [5] derived condition under which $\left(T^{*}, F^{*}\right)$ gives an optimal solution; that is,

$$
\beta \geq \beta^{*}=1-\sqrt{\frac{2 C_{0} C_{h}^{\prime}}{D C_{1}^{2}}},
$$

where $\beta^{*}$ denotes the critical value of the backordering rate. Then, Pentico et al. [5] determine the optimal inventory policy in a way that if condition (4) is satisfied, then the optimal policy is to allow stockouts with partial backordering and meet the fractional demand; otherwise the optimal policy is either to meet all demand or to lose all sales. When condition (4) is satisfied, Pentico et al. [5] proved the optimality of the solution for the EPQ-PBO given with (2) and (3), by examining the characteristics of the partial derivatives and the boundary conditions. In this paper, we are going to prove the optimality of the solution by using the mathematical programming approach.

Recognition of the (global) optimal solution for the general nonlinear programming problem using the nonlinear programming theory is not an easy task. There are sufficient conditions for the local optimality, but there are very few or too restrictive for the global optimality. Among the inventory models there are many with a convex objective function, but most problems arise when the objective function is nonconvex, because otherwise the local optimal solution is global. In this case, with the nonconvex objective, proving the global optimality of the solution for the model is usually done by examining the partial derivatives and the boundary conditions; some of them are $[2,5,9,10]$. There are some global optimality theories for general nonlinear programming problems, that are quite effective in practice for certain classes of problems. One of them is the canonical duality and trivial theory proposed by Gao and Sherali, which has good performance for quadratic programming problems in mechanics [11]. Algebraical approach is another approach that is used with inventory models; see [12,13]. Proving the optimality of the solution becomes more complex when there is some uncertainty in the inventory system; see $[14,15]$.

The paper is organized as follows. In Section 2 Pentico et al.s EPQ-PBO is formulated as a mathematical programming problem, then mathematical programming approach for proving the optimality of the solution is presented, and the same approach is used for two other inventory models with partial backordering, an extension of Pentico et al's EPQ$\mathrm{PBO}$ [6] and $\mathrm{Hu}$ et al.s inventory model [2]. There are comments on how mathematical programming approach can be used for the models presented in Taleizadeh et al. [16], Taleizadeh et al. [10], Taleizadeh and Pentico [9], and Cárdenas-Barrón [12].

\section{Mathematical Programming Approach}

2.1. Formulation as a Mathematical Programming Problem. For the decision variables, the length of an order cycle $T$ and the fill rate $F$, there are natural restrictions; that is, the following conditions should be met:

$$
T>0, \quad 0 \leq F \leq 1 .
$$

So, the corresponding mathematical programming problem for Pentico et al.s EPQ-PBO [5] is a box-constrained nonlinear programming problem and is formulated as follows:

$$
\begin{aligned}
& \min _{(T, F) \in \mathbb{R}^{2}} \Gamma(T, F) \\
& \text { subject to } T>0,0 \leq F \leq 1,
\end{aligned}
$$

where $\Gamma(T, F)$ is given with (1). Denote the feasible region for the problem (6) by $\mathscr{F}=\left\{(T, F) \in \mathbb{R}^{2} \mid T>0,0 \leq F \leq 1\right\}$. We should note that, in their later work on the extension of the EPQ-PBO, [1], the authors impose these conditions on the decision variables $T$ and $F$ and formulated the corresponding mathematical programming problem, and for the proof of the optimality of the solution they still have the same approach as that for the basic EPQ-PBO [5].

In the next subsection, using the nonlinear programming theory, we are going to prove that the solution $\left(T^{*}, F^{*}\right)$ given by (2) and (3) is a global minimizer of the problem (6), if the condition on the backordering rate, that is, $\beta \geq \beta^{*}$, is satisfied. We will also make some comments on choosing the optimal policy among all three policies: to meet fractional demand, to meet all demand, or to lose all sales, as a consequence of solving the problem (6) via nonlinear programming theory.

2.2. Proving the Optimality of the Solution. First, let us note that the equivalent form of the condition $\beta \geq \beta^{*}$, where $\beta^{*}$ is the critical value of the backordering rate from (4), is the following inequality:

$$
\frac{2 C_{0}}{D C_{h}^{\prime}} \geq \frac{\left(C_{1}^{\prime}\right)^{2}}{\left(C_{h}^{\prime}\right)^{2}}
$$

With the following two lemmas, we will prove that under the condition $\beta \geq \beta^{*}$, the solution $\left(T^{*}, F^{*}\right)$ given by (2) and (3) is well defined (Lemma 1 ) and is a feasible solution for the problem (6) (Lemma 2).

Lemma 1. If $\beta \geq \beta^{*}$, then $\left(T^{*}, F^{*}\right)$ is well defined. 
Proof. Assume that $\beta \geq \beta^{*}$. Then, using inequality (7), for the expression under the square root in the formula (2) we have

$$
\begin{aligned}
& \frac{2 C_{0}}{D C_{h}^{\prime}}\left(\frac{C_{h}^{\prime}+\beta C_{b}^{\prime}}{\beta C_{b}^{\prime}}\right)-\frac{\left(C_{1}^{\prime}\right)^{2}}{\beta C_{h}^{\prime} C_{b}^{\prime}} \\
& \quad \geq \frac{\left(C_{1}^{\prime}\right)^{2}}{\left(C_{h}^{\prime}\right)^{2}}\left(\frac{C_{h}^{\prime}+\beta C_{b}^{\prime}}{\beta C_{b}^{\prime}}\right)-\frac{\left(C_{1}^{\prime}\right)^{2}}{\beta C_{h}^{\prime} C_{b}^{\prime}} \\
& \quad=\frac{\left(C_{1}^{\prime}\right)^{2}}{\left(C_{h}^{\prime}\right)^{2}}\left(\frac{C_{h}^{\prime}+\beta C_{b}^{\prime}}{\beta C_{b}^{\prime}}-\frac{C_{h}^{\prime}}{\beta C_{b}^{\prime}}\right)=\frac{\left(C_{1}^{\prime}\right)^{2}}{\left(C_{h}^{\prime}\right)^{2}} \geq 0
\end{aligned}
$$

so $T^{*}$ is well defined, which implies that $F^{*}$ is also well defined.

Lemma 2. $\beta \geq \beta^{*}$ if and only if $F^{*} \leq 1$.

Proof. Using (2), (3), and (7) we have

$$
\begin{aligned}
F^{*} \leq 1 & \Longleftrightarrow \frac{C_{1}^{\prime}+\beta C_{b}^{\prime} T^{*}}{T^{*}\left(C_{h}^{\prime}+\beta C_{b}^{\prime}\right)} \leq 1 \\
& \Longleftrightarrow C_{1}^{\prime}+\beta C_{b}^{\prime} T^{*} \leq T^{*}\left(C_{h}^{\prime}+\beta C_{b}^{\prime}\right) \\
& \Longleftrightarrow T^{*} \geq \frac{C_{1}^{\prime}}{C_{h}^{\prime}} \\
& \Longleftrightarrow \frac{2 C_{0}}{D C_{h}^{\prime}}\left(\frac{C_{h}^{\prime}+\beta C_{b}^{\prime}}{\beta C_{b}^{\prime}}\right)-\frac{\left(C_{1}^{\prime}\right)^{2}}{\beta C_{h}^{\prime} C_{b}^{\prime}} \geq \frac{\left(C_{1}^{\prime}\right)^{2}}{\left(C_{h}^{\prime}\right)^{2}} \\
& \Longleftrightarrow \frac{2 C_{0}}{D C_{h}^{\prime}}\left(\frac{C_{h}^{\prime}+\beta C_{b}^{\prime}}{\beta C_{b}^{\prime}}\right) \geq \frac{\left(C_{1}^{\prime}\right)^{2}}{\left(C_{h}^{\prime}\right)^{2}}\left(\frac{C_{h}^{\prime}}{\beta C_{b}^{\prime}}+1\right) \\
& \Longleftrightarrow \frac{2 C_{0}}{D C_{h}^{\prime}}\left(\frac{C_{h}^{\prime}+\beta C_{b}^{\prime}}{\beta C_{b}^{\prime}}\right) \geq \frac{\left(C_{1}^{\prime}\right)^{2}}{\left(C_{h}^{\prime}\right)^{2}}\left(\frac{C_{h}^{\prime}+\beta C_{b}^{\prime}}{\beta C_{b}^{\prime}}\right) \\
& \Longleftrightarrow \frac{2 C_{0}}{D C_{h}^{\prime}} \geq \frac{\left(C_{1}^{\prime}\right)^{2}}{\left(C_{h}^{\prime}\right)^{2}} \Longleftrightarrow \beta \geq \beta \beta^{*},
\end{aligned}
$$

which completes the proof.

So, if $\beta \geq \beta^{*}$, we have that $\left(T^{*}, F^{*}\right)$ is well defined (Lemma 1), and because it is obvious that $T^{*}>0$ and consequently $F^{*}>0$ and we have that $F^{*} \leq 1$ (Lemma 2 ), we can finally conclude that $\left(T^{*}, F^{*}\right) \in \mathscr{F}$; that is, $\left(T^{*}, F^{*}\right)$ is a feasible point for the problem (6). Since the statement in Lemma 2 is true in both directions, the value $\beta^{*}$ can also be considered as a critical value for the feasibility of the solution. This is why when $\beta<\beta^{*}$, due to the fact that in that case the solution $\left(T^{*}, F^{*}\right)$ is not feasible, the policy of meeting the fractional demand cannot be optimal, and we should decide between meeting all demand or losing all sales, as Pentico et al. [5] proposed. But when $\beta<\beta^{*}$, the optimal policy is to meet all demand for sure, as it is shown by Stojkovska [17].
In the remaining part of this subsection, we will prove the optimality of the solution $\left(T^{*}, F^{*}\right)$ by examining the optimality conditions for the problem (6). We will use the optimality conditions that deal with the linear independenceconstrained qualification (LICQ), that is, LICQ holds at a point if the set of active constrained gradients in this point is linearly independent, and the KKT point, that is, the point for which there are Lagrangian multipliers such that the KKT system is satisfied, which are not always easily verified; see, for example [18]. But, the problem under our consideration (6) has the suitable form for checking these conditions, as we will demonstrate.

To prove the optimality of the solution $\left(T^{*}, F^{*}\right)$, we will first find all KKT points for the problem (6) (Proposition 3). Then, we will prove that $\left(T^{*}, F^{*}\right)$ is a local minimizer using the second-order sufficient conditions, and the global optimality of $\left(T^{*}, F^{*}\right)$ will be a consequence of the previous findings (Proposition 4).

Proposition 3. Assume that $\beta \geq \beta^{*}$, where $\beta^{*}$ is defined by (4). Then $\left(T^{*}, F^{*}\right)$ defined by (2) and (3) is the only KKT point for the problem (6).

The KKT point for the problem (6) lies on the boundary $F=1$ if and only if $\beta=\beta^{*}$.

Proof. The problem (6) has three constraints $T>0, F \geq 0$, and $1-F \geq 0$. So, the corresponding KKT system for the problem $(6)$ is

$$
\begin{gathered}
\frac{\partial \Gamma}{\partial T}-\lambda_{1}=0, \quad \frac{\partial \Gamma}{\partial F}-\lambda_{2}+\lambda_{3}=0, \\
T>0, \quad F \geq 0, \quad 1-F \geq 0, \\
\lambda_{1} T=0, \quad \lambda_{2} F=0, \quad \lambda_{3}(1-F)=0, \\
\lambda_{1} \geq 0, \quad \lambda_{2} \geq 0, \quad \lambda_{3} \geq 0,
\end{gathered}
$$

where $\lambda_{1}, \lambda_{2}$, and $\lambda_{3}$ are the Lagrangian multipliers, and the first partial derivatives are

$$
\begin{aligned}
& \frac{\partial \Gamma}{\partial T}=-\frac{C_{0}}{T^{2}}+\frac{C_{h}^{\prime} D F^{2}}{2}+\frac{\beta C_{b}^{\prime} D(1-F)^{2}}{2}, \\
& \frac{\partial \Gamma}{\partial F}=C_{h}^{\prime} D T F-\beta C_{b}^{\prime} D T(1-F)-C_{1}^{\prime} D .
\end{aligned}
$$

To obtain all KKT points we need to solve the KKT system (10)-(13) with respect to $T, F, \lambda_{1}, \lambda_{2}$, and $\lambda_{3}$. Note that one easy approach for solving the KKT system is to start with complementarity conditions (12). Because $T>0$, from the first complementarity condition in (12), we have $\lambda_{1}=0$, and from the first equation in (10), we have $\partial \Gamma / \partial T=0$.

Assume that $F=0$. Then, from the third complementarity condition in (12), we have $\lambda_{3}=0$, and from the second equation in (10), we have $\partial \Gamma / \partial F-\lambda_{2}=0$. Substituting $F=0$ in the last equation, we have $\lambda_{2}=\partial \Gamma / \partial F=-\beta C_{b}^{\prime} D T-C_{1}^{\prime} D<0$, with its strict inequality sign, since $T>0$. This, contradicts with the second inequality in (13). So, it must be that $F>0$.

Now, from $F>0$, and the second complementarity condition in (12), we have $\lambda_{2}=0$. 
Assume that $\lambda_{3}=0$. Then, from the second equation in (10), we have $\partial \Gamma / \partial F=0$. Recall that $\left(T^{*}, F^{*}\right)$ is the solution obtained by setting the first partial derivatives to zero and taking the positive value for $T$. Now, from Lemma 2 , when $\beta \geq \beta^{*},\left(T^{*}, F^{*}\right)$ satisfies the feasibility conditions (11). We obtained that, for $\lambda_{1}=\lambda_{2}=\lambda_{3}=0,\left(T^{*}, F^{*}\right)$ satisfies the KKT system, so $\left(T^{*}, F^{*}\right)$ is a KKT point.

What is left, to completely solve the KKT system (10)(13), is to assume that $F=1$. Then, substituting $F=1$ into $\partial \Gamma / \partial T=0$, we have $T^{2}=2 C_{0} / C_{h}^{\prime} D$, and $T=\sqrt{2 C_{0} / C_{h}^{\prime} D}>0$ is the only positive solution. Now, from the second equation in (10) and $\lambda_{2}=0$, we have $\lambda_{3}=-\partial \Gamma / \partial F=-C_{h}^{\prime} D T+C_{1}^{\prime} D=$ $-C_{h}^{\prime} D \sqrt{2 C_{0} / C_{h}^{\prime} D}+C_{1}^{\prime} D=C_{h}^{\prime} D\left(C_{1}^{\prime} / C_{h}^{\prime}-\sqrt{2 C_{0} / C_{h}^{\prime} D}\right)$. Having in mind that (7) is equivalent to $\beta \geq \beta^{*}$, we have that $\lambda_{3} \leq 0$ if and only if $\beta \geq \beta^{*}$. But from the third inequality in (13), we have that $\lambda_{3}=0$, and this is true if and only if $\beta=\beta^{*}$. Since all three Lagrangian multipliers in this case are zero $\lambda_{1}=\lambda_{2}=\lambda_{3}=0$, the newly found solution is actually the solution $\left(T^{*}, F^{*}\right)$ when $\beta=\beta^{*}$, in which case we proved that it lies on the boundary $F=1$.

So, if $\beta \geq \beta^{*}$, then $\left(T^{*}, F^{*}\right)$ is the unique KKT point, that lies on the boundary $F=1$ if and only if $\beta=\beta^{*}$, which we wanted to prove.

Proposition 4. Assume that $\beta \geq \beta^{*}$, where $\beta^{*}$ is defined by (4). Then $\left(T^{*}, F^{*}\right)$ defined by (2) and (3) is a local minimizer of the problem (6).

Moreover, if $\beta \geq \beta^{*}$, then $\left(T^{*}, F^{*}\right)$ is the global minimizer of the problem (6).

Proof. Assume that $\beta \geq \beta^{*}$. From Proposition 3 we have that $\left(T^{*}, F^{*}\right)$ is a KKT point for the problem (6). We are going to prove that the Hessian matrix of the Lagrangian calculated at the point $\left(T^{*}, F^{*}\right)$ is positive definite.

For the problem (6), the Hessian matrix of the Lagrangian coincides with the Hessian matrix of the objective function; that is,

$$
H(T, F)=\left[\begin{array}{cc}
\frac{2 C_{0}}{T^{3}} & C_{h}^{\prime} D F-\beta C_{b}^{\prime} D(1-F) \\
C_{h}^{\prime} D F-\beta C_{b}^{\prime} D(1-F) & C_{h}^{\prime} D T+\beta C_{b}^{\prime} D T
\end{array}\right] .
$$

Now, let us examine the signs of the principals of the above Hessian calculated at $\left(T^{*}, F^{*}\right)$. But first, let us make some observations. We know that the first partial derivative with respect to $F$ calculated at the solution $\left(T^{*}, F^{*}\right)$ is zero; that is,

$$
C_{h}^{\prime} D T^{*} F^{*}-\beta C_{b}^{\prime} D T^{*}\left(1-F^{*}\right)-C_{1}^{\prime} D=0 .
$$

From the last equality we obtain the following one:

$$
T^{*}\left(C_{h}^{\prime} F^{*}-\beta C_{b}^{\prime}\left(1-F^{*}\right)\right)=C_{1}^{\prime} .
$$

Now, for the first principal of the above Hessian we have

$$
H\left(T^{*}, F^{*}\right)_{11}=\frac{2 C_{0}}{\left(T^{*}\right)^{3}}>0 .
$$

And for the second principal by using (7) and (17) we have

$$
\begin{aligned}
& H\left(T^{*}, F^{*}\right)_{22} \\
& =\left|\begin{array}{cc}
\frac{2 C_{0}}{\left(T^{*}\right)^{3}} & C_{h}^{\prime} D F^{*}-\beta C_{b}^{\prime} D\left(1-F^{*}\right) \\
C_{h}^{\prime} D F^{*}-\beta C_{b}^{\prime} D\left(1-F^{*}\right) & C_{h}^{\prime} D T^{*}+\beta C_{b}^{\prime} D T^{*}
\end{array}\right| \\
& =\frac{2 C_{0}}{\left(T^{*}\right)^{3}}\left(C_{h}^{\prime} D T^{*}+\beta C_{b}^{\prime} D T^{*}\right) \\
& -\left(C_{h}^{\prime} D F^{*}-\beta C_{b}^{\prime} D\left(1-F^{*}\right)\right)^{2} \\
& =\frac{D}{\left(T^{*}\right)^{2}}\left(2 C_{0}\left(C_{h}^{\prime}+\beta C_{b}^{\prime}\right)\right. \\
& \left.-D\left(T^{*}\right)^{2}\left(C_{h}^{\prime} F^{*}-\beta C_{b}^{\prime}\left(1-F^{*}\right)\right)^{2}\right) \\
& =\frac{D}{\left(T^{*}\right)^{2}}\left(2 C_{0}\left(C_{h}^{\prime}+\beta C_{b}^{\prime}\right)-D\left(C_{1}^{\prime}\right)^{2}\right) \\
& \geq \frac{D}{\left(T^{*}\right)^{2}}\left(2 C_{0}\left(C_{h}^{\prime}+\beta C_{b}^{\prime}\right)-D \frac{2 C_{0} C_{h}^{\prime}}{D}\right) \\
& =\frac{D}{\left(T^{*}\right)^{2}} 2 C_{0} \beta C_{b}^{\prime}
\end{aligned}
$$

$>0$.

Both principals of the Hessian calculated at $\left(T^{*}, F^{*}\right)$ are positive, so the Hessian calculated at $\left(T^{*}, F^{*}\right)$ is a positive definite matrix. This together with the fact that $\left(T^{*}, F^{*}\right)$ is a KKT point for the problem (6) when $\beta \geq \beta^{*}$ implies that $\left(T^{*}, F^{*}\right)$ is a local minimizer of the problem (6), when $\beta \geq \beta^{*}$.

Let us note that for the box-constrained problem (6), LICQ holds at every feasible point. So, if there is another local minimizer $(\widehat{T}, \widehat{F})$ for the problem (6) different from $\left(T^{*}, F^{*}\right)$, then $(\widehat{T}, \widehat{F})$ is also a KKT point for the problem (6), which contradicts with the uniqueness of the KKT point $\left(T^{*}, F^{*}\right)$ (Proposition 3). This implies that $\left(T^{*}, F^{*}\right)$ is the global minimizer for the problem (6), which completes the proof.

It can be seen from the proof of Proposition 3 that when the solution $\left(T^{*}, F^{*}\right)$ lies on the boundary $F=1$, then it coincides with the basic EPQ solution, which is always on the boundary $F=1$. Since $\left(T^{*}, F^{*}\right)$ minimizes the cost function $\Gamma(T, F)$ when $\beta \geq \beta^{*}$ (Proposition 4 ), the partial backordering policy is optimal compared to the policy of meeting all demand, when $\beta \geq \beta^{*}$. But as Zhang [19] noticed, when $\beta \geq \beta^{*}$, it is not always preferable to meet the fractional demand; losing all sales can actually be a better decision. Later, the proposed condition by Zhang, under which meeting the fractional demand is optimal compared to losing all sales when $\beta \geq \beta^{*}$, is corrected by Stojkovska [17].

If the production rate is infinitely large, the Pentico et al's EPQ-PBO [5] will degenerate into Pentico and Drake's EOQPBO [4]. When we substitute $C_{h}$ for $C_{h}^{\prime}, C_{b}$, for $C_{b}^{\prime}$ the above propositions can be used for Pentico and Drake's EOQ-PBO [4]. 
2.3. Implementation to Other Inventory Models with Partial Backordering. It is difficult to use the above mathematical programming approach as a general solution procedure, because its implementation depends on finding all KKT points for the corresponding mathematical programming problem and examining the Hessian in the candidate solution. But many inventory models are suitable for this approach. Here, we consider two more models.

2.3.1. Implementation to an Extension of Pentico et al.'s EPQ$P B O$ [6]. One of the extensions to the Pentico et al.s EPQ$\mathrm{PBO}$ is the EPQ-PBO and phase-dependent backordering rate [6]. Relaxing the assumption on a constant backordering rate $\beta$, they considered two phases of constant backordering rate. During the first phase, before the start of the production, the backordering rate is $\beta$, and during the second phase, after the production starts, the backordering rate is $\rho \beta$, where $1 \leq$ $\rho \leq 1 / \beta$. For this extension by using a different methodology, the arithmetic-geometric-mean-inequality approach, Hsieh and Dye [13] derived the solution and proved its optimality. We will prove the optimality of the solution for this extension with the mathematical programming approach.

For this model, the total cost function $\Gamma(T, F)$, the optimal length of an order cycle $T^{*}$, and the optimal fill rate $F^{*}$ are of the same form as the ones for the EPQ-PBO given with the formulas (1), (2), and (3), respectively, where $C_{h}^{\prime}=$ $C_{h}(1-D / P), C_{b}^{\prime}=\left(C_{b}(1-\rho \beta D / P)\right) /(1-(\rho-1) \beta D / P)$, and $C_{1}^{\prime}=C_{1}(1-\beta /(1-(\rho-1) \beta D / P))$. The critical value of the backordering rate $\beta$ developed by Pentico et al. [6], which we will denote by $\beta_{\text {new }}^{*}$, is

$$
\beta_{\text {new }}^{*}=\frac{\beta^{*}}{1+(\rho-1)(D / P) \beta^{*}},
$$

where $\beta^{*}$ is defined by (4). Pentico et al. [6] proved that if $\beta \geq \beta_{\text {new }}^{*}$, then $\left(T^{*}, F^{*}\right)$ is the optimal solution. They based the proof of the optimality on the same approach presented for the basic EPQ-PBO [5]. We will use the mathematical programming approach for proving the optimality of the solution for this extension.

First, let us notice that the equivalent form of the condition $\beta \geq \beta_{\text {new }}^{*}$, where $\beta_{\text {new }}^{*}$ is defined by (20), is the condition of the form (7), where $C_{h}^{\prime}=C_{h}(1-D / P)$ and $C_{1}^{\prime}=C_{1}(1-$ $\beta /(1-(\rho-1) \beta D / P))$ are notations that correspond to the extension. Therefore, the proof that the solution $\left(T^{*}, F^{*}\right)$ is well defined and feasible under condition $\beta \geq \beta_{\text {new }}^{*}$ imitates the proofs of Lemmas 1 and 2 for the solution of the basic EPQ-PBO from the previous subsection. For the EPQ-PBO and phase-dependent backordering rate it can be also proven in a same way, as in Propositions 3 and 4 , that if $\beta \geq \beta_{\text {new }}^{*}$, $\left(T^{*}, F^{*}\right)$ is the only KKT point which lies on the boundary $F=1$ if and only if $\beta=\beta_{\text {new }}^{*}$, and when $\beta \geq \beta_{\text {new }}^{*}$, then $\left(T^{*}, F^{*}\right)$ is the global minimizer of the objective $\Gamma(T, F)$.

The same approach can be used for other extensions of Pentico and Drake's basic EOQ-PBO, [4], or Pentico et al.s basic EPQ-PBO, [5]. One of the most recent extensions of Pentico and Drake's basic EOQ-PBO [4] is models presented by Taleizadeh et al. [16], Taleizadeh et al. [10], and Taleizadeh and Pentico [9]. In these papers, the proof of the optimality of the solution is made using the same methodology as in [4], since the objective function has the similar form. So, the mathematical programming approach presented here can also be implemented for these inventory models in a similar way. It can be shown that when some condition on the backordering rate (and a condition on the price increase for the model presented in [9]) is met, then the corresponding mathematical programming problem has a unique KKT point which is the global optimizer of the objective function. When condition on the backordering rate is satisfied with its equality part, then the optimal point lies on the boundary.

2.3.2. Implementation to Hu et al.'s Inventory Model, [2]. Hu et al. [2] proposed an EOQ-PBO inventory model with constant backordering rate and unit backorder cost increasing linearly with the duration of the shortage. Their decision variables are the cycle time $T$ and the time to which the inventory is positive $t_{1}$. In their notation, the total cost per unit time that has to be minimized is

$$
\begin{aligned}
\operatorname{TRC}\left(T, t_{1}\right)=\frac{1}{T}(A & +\frac{C i t_{1}^{2} \alpha}{2} \\
& +r \alpha\left(\frac{b}{6}\left(T-t_{1}\right)^{3}+\frac{C_{0}}{2}\left(T-t_{1}\right)^{2}\right) \\
& \left.+R(1-r) \alpha\left(T-t_{1}\right)\right),
\end{aligned}
$$

and the minimization is performed under the constrains $T>$ $0,0 \leq t_{1} \leq T$. Then, Hu et al. determine the optimal solution by approximately solving by a numerical search an equation with respect to $t_{1}$ and the obtained value they substitute in an equation for $T$. They established a critical value for the holding cost per unit, and by examining the partial derivatives and boundary conditions, they proved that if the condition

$$
C i \geq \frac{R^{2}(1-r)^{2} \alpha}{2 A}
$$

is satisfied, then partial backordering is optimal; otherwise, the optimal policy is not to allow backordering. We are going to find the solution and prove its optimality by the mathematical programming approach.

If we put $t_{1}=F T$, where $0 \leq F \leq 1$ is the fill rate, then we can transform the total cost function into

$$
\begin{aligned}
\operatorname{TRC}(T, F)= & \frac{A}{T}+\frac{C i F^{2} T \alpha}{2}+\frac{r \alpha b}{6} T^{2}(1-F)^{3} \\
& +\frac{r \alpha C_{0}}{2} T(1-F)^{2}+R(1-r) \alpha(1-F) .
\end{aligned}
$$

The corresponding mathematical programming problem is

$$
\begin{aligned}
& \min _{(T, F) \in \mathbb{R}^{2}} \operatorname{TRC}(T, F) \\
& \text { subject to } T>0,0 \leq F \leq 1,
\end{aligned}
$$

where $\operatorname{TRC}(T, F)$ is given with (23). We should note that the introduction of new decision variables was not needed to 
demonstrate the mathematical programming approach and prove the optimality of the solution. This transformation is done mainly to shorten the proofs related to this model and to be able to lean on the ones for Pentico et al.s EPQ-PBO presented in Section 2.2.

First partial derivatives of $\operatorname{TRC}(T, F)$ with respect to $T$ and $F$, respectively, are

$$
\begin{aligned}
\frac{\partial \operatorname{TRC}(T, F)}{\partial T}= & -\frac{A}{T^{2}}+\frac{C i F^{2} \alpha}{2}+\frac{r \alpha b}{3} T(1-F)^{3} \\
& +\frac{r \alpha C_{0}}{2}(1-F)^{2}, \\
\frac{\partial \operatorname{TRC}(T, F)}{\partial F}= & C i F T \alpha-\frac{r \alpha b}{2} T^{2}(1-F)^{2} \\
& -r \alpha C_{0} T(1-F)-R(1-r) \alpha .
\end{aligned}
$$

If we solve $(\partial \operatorname{TRC}(T, F)) / \partial F=0$ with respect to $F, 0 \leq F \leq 1$, we will have

$$
\begin{aligned}
F & =F(T) \\
& =1-\frac{-\left(r C_{0}+C i\right)+\sqrt{\left(r C_{0}+C i\right)^{2}-4 r b(R(1-r)-C i T)}}{r b T} .
\end{aligned}
$$

If we substitute the expressions for $(1-F) T$ and $F T$ that are derived form $(26)$ in $(\partial \operatorname{TRC}(T, F)) / \partial T=0$, we will have

$$
\frac{C i \alpha}{2}(T-\varphi(T))^{2}+\frac{r \alpha b}{3} \varphi(T)^{3}+\frac{r \alpha C_{0}}{2} \varphi(T)^{2}=A,
$$

where $\varphi(T) \quad=\quad\left(-\left(r C_{0}+C i\right)+\right.$ $\left.\sqrt{\left(r C_{0}+C i\right)^{2}-4 r b(R(1-r)-C i T)}\right) /(r b)$. Let us denote by $T^{*}$ the solution of (27) and by $F^{*}=F\left(T^{*}\right)=1-\varphi\left(T^{*}\right) / T^{*}$.

Under the condition (22), (27) has the unique solution $T^{*}$ on $T>R(1-r) /(C i)$, since the function on the left side of (27) is nondecreasing with respect to $T$, with a value less or equal to $A$ at $R(1-r) /(C i)$ (from where the condition (22) is derived), and infinity, when $T \rightarrow \infty$. And since the expression under the square root in (26) is positive on $T>$ $R(1-r) /(C i)$, we have that when the condition (22) is satisfied, the solution $\left(T^{*}, F^{*}\right)$ is well defined and unique on $T>R(1-$ $r) /(C i)$.

From the equivalence $F^{*} \leq 1 \Leftrightarrow T^{*} \geq R(1-r) /(C i)$, we have that if the condition (22) is satisfied, then the solution $\left(T^{*}, F^{*}\right)$ is well defined, unique, and feasible for the problem (24) on $T>R(1-r) /(C i)$. Otherwise, if condition (22) is not satisfied, then (27) does not have the solution on $T>$ $R(1-r) /(C i)$, which implies that in that case the partial backordering solution $\left(T^{*}, F^{*}\right)$ is not feasible ( since $F^{*}>1$ ), and in that case the optimal policy is to meet all demand according to the EOQ model with no shortage period with optimal time cycle $T_{\mathrm{EOQ}}^{*}=\sqrt{(2 A) /(\mathrm{Ci \alpha})}$.

Similarly to Proposition 3, it can be shown that when the condition (22) is satisfied, then $\left(T^{*}, F^{*}\right)$ is the only KKT point for the problem (24), which lies on the boundary $F=1$ when the condition (22) is satisfied with equality sign and in that case the solution $\left(T^{*}, F^{*}\right)$ coincides with the solution from the EOQ model with no shortages. And finally, similarly to Proposition 4, it can be shown that when the condition (22) is satisfied, then the Hessian calculated at $\left(T^{*}, F^{*}\right)$ is positive definite, so $\left(T^{*}, F^{*}\right)$ is the local minimizer, and due to its uniqueness as a KKT point, it is the global minimizer for the problem (24).

This approach of transforming the mathematical programming problem into decision variables $T$ and $F$ can be used within other models, for example, the EPQ model with full backordering considered by Cárdenas-Barrón [12]. After transformation it can be proven that there is a unique KKT point, and it lies in the interior of the feasible region, and at this point the Hessian matrix is positive definite, which implies the optimality of the solution.

\section{Conclusions}

In this paper, we give an alternative way of deriving the solution for the Pentico et al.s EPQ-PBO and proving its optimality, by using the mathematical programming approach. We also determine the optimal decision policy among meeting fractional demand, meeting all demand, and losing all sales, while solving the corresponding mathematical programming problem. We use the same approach for two other inventory models with partial backordering. If we can find all KKT points for the mathematical programming problem, then this approach can be used for deriving and proving optimality of the solution for the corresponding inventory model.

\section{References}

[1] M. J. Drake, D. W. Pentico, and C. Toews, "Using the EPQ for coordinated planning of a product with partial backordering and its components," Mathematical and Computer Modelling, vol. 53, no. 1-2, pp. 359-375, 2011.

[2] W. Hu, S. L. Kim, and A. Banerjee, "An inventory model with partial backordering and unit backorder cost linearly increasing with the waiting time," European Journal of Operational Research, vol. 197, no. 2, pp. 581-587, 2009.

[3] Z. Pang, "Optimal dynamic pricing and inventory control with stock deterioration and partial backordering," Operations Research Letters, vol. 39, no. 5, pp. 375-379, 2011.

[4] D. W. Pentico and M. J. Drake, "The deterministic EOQ with partial backordering: a new approach," European Journal of Operational Research, vol. 194, no. 1, pp. 102-113, 2009.

[5] D. W. Pentico, M. J. Drake, and C. Toews, "The deterministic EPQ with partial backordering: a new approach," Omega, vol. 37, no. 3, pp. 624-636, 2009.

[6] D. W. Pentico, M. J. Drake, and C. Toews, "The EPQ with partial backordering and phase-dependent backordering rate," Omega, vol. 39, no. 5, pp. 574-577, 2011.

[7] H.-L. Yang, "A partial backlogging inventory model for deteriorating items with fluctuating selling price and purchasing cost," Advances in Operations Research, vol. 2012, Article ID 385371, 15 pages, 2012.

[8] D. W. Pentico and M. J. Drake, "A survey of deterministic models for the EOQ and EPQ with partial backordering," European Journal of Operational Research, vol. 214, no. 2, pp. 179-198, 2011. 
[9] A. A. Taleizadeh and D. W. Pentico, "An economic order quantity model with a known price increase and partial backordering," European Journal of Operational Research, vol. 228, no. 3, pp. 516-525, 2013.

[10] A. A. Taleizadeh, D. W. Pentico, M. S. Jabalameli, and M. Aryanezhad, "An economic order quantity model with multiple partial prepayments and partial backordering," Mathematical and Computer Modelling, vol. 57, no. 3-4, pp. 311-323, 2013.

[11] D. Y. Gao and H. D. Sherali, "Canonical duality theory: connection between nonconvex mechanics and global optimization," in Advances in Mechanics and Mathematics, vol. 3, pp. 249-316, Springer, 2007.

[12] L. E. Cárdenas-Barrón, “The economic production quantity (EPQ) with shortage derived algebraically," International Journal of Production Economics, vol. 70, no. 3, pp. 289-292, 2001.

[13] T. P. Hsieh and C. Y. Dye, "A note on 'The EPQ with partial backordering and phase-dependent backordering rate,' Omega, vol. 40, no. 1, pp. 131-133, 2012.

[14] Y. Zhao and M. N. Katehakis, "On the structure of optimal ordering policies for stochastic inventory systems with minimum order quantity," Probability in the Engineering and Informational Sciences, vol. 20, no. 2, pp. 257-270, 2006.

[15] B. Zhou, Y. Zhao, and M. N. Katehakis, "Effective control policies for stochastic inventory systems with a minimum order quantity and linear costs," International Journal of Production Economics, vol. 106, no. 2, pp. 523-531, 2007.

[16] A. A. Taleizadeh, D. W. Pentico, M. Aryanezhad, and S. M. Ghoreyshi, "An economic order quantity model with partial backordering and a special sale price," European Journal of Operational Research, vol. 221, no. 3, pp. 571-583, 2012.

[17] I. Stojkovska, "On the optimality of the optimal policies for the deterministic EPQ with partial backordering," Omega, vol. 41, no. 5, pp. 919-923, 2013.

[18] J. Nocedal and S. J. Wright, Numerical Optimization, Springer, New York, NY, USA, 1999.

[19] R. Q. Zhang, "A note on the deterministic EPQ with partial backordering," Omega, vol. 37, no. 5, pp. 1036-1038, 2009. 


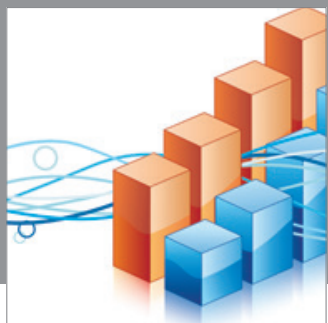

Advances in

Operations Research

mansans

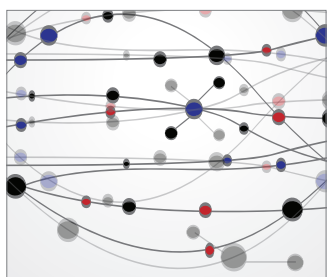

The Scientific World Journal
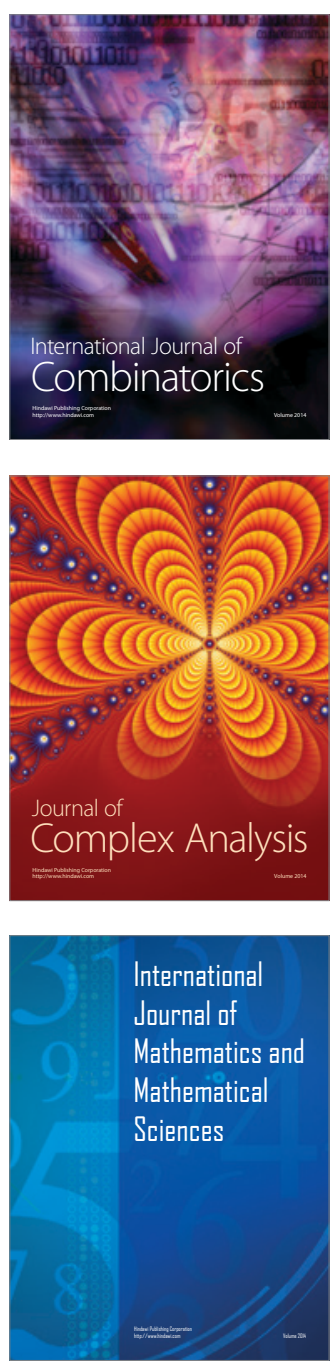
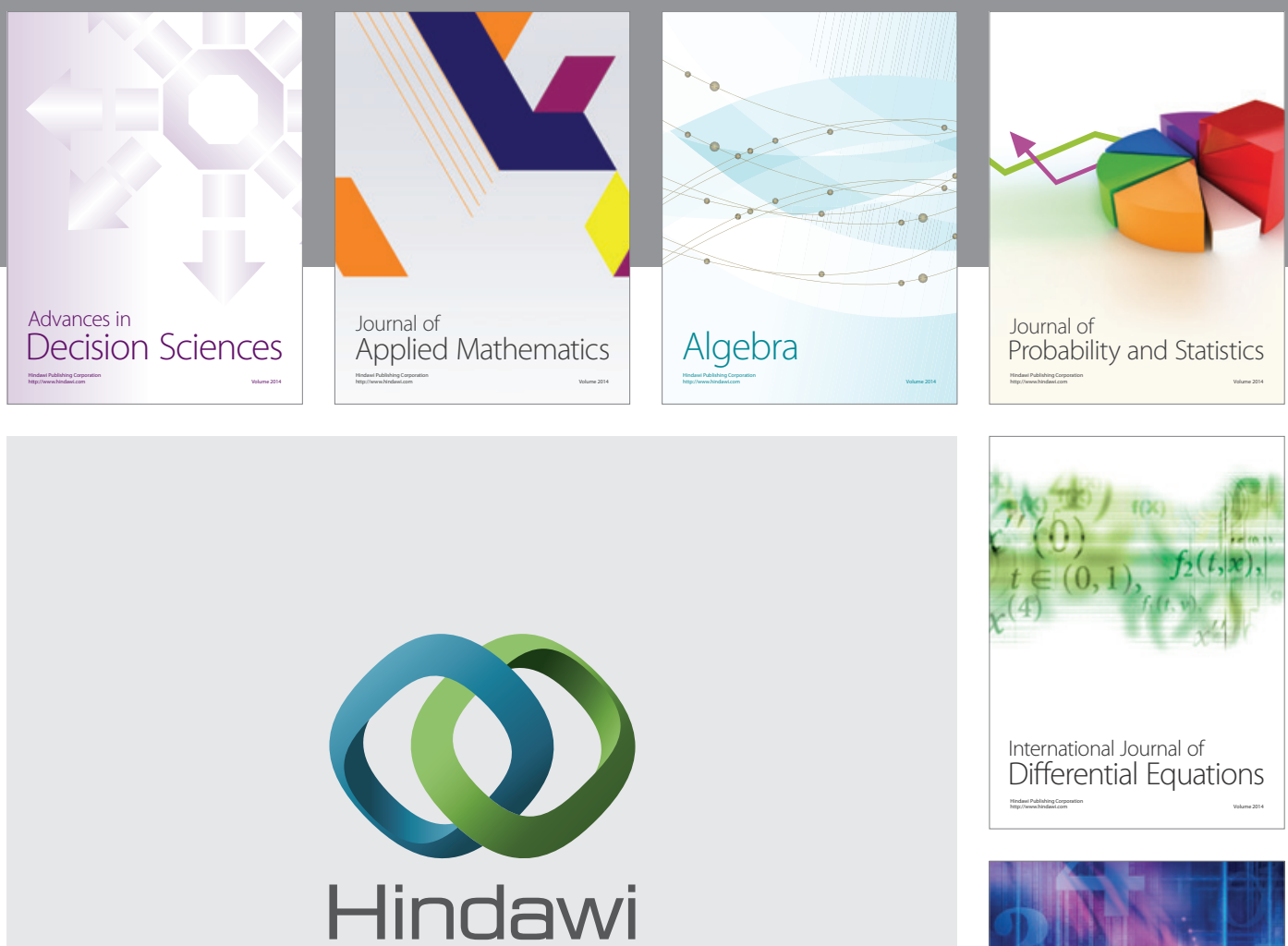

Submit your manuscripts at http://www.hindawi.com
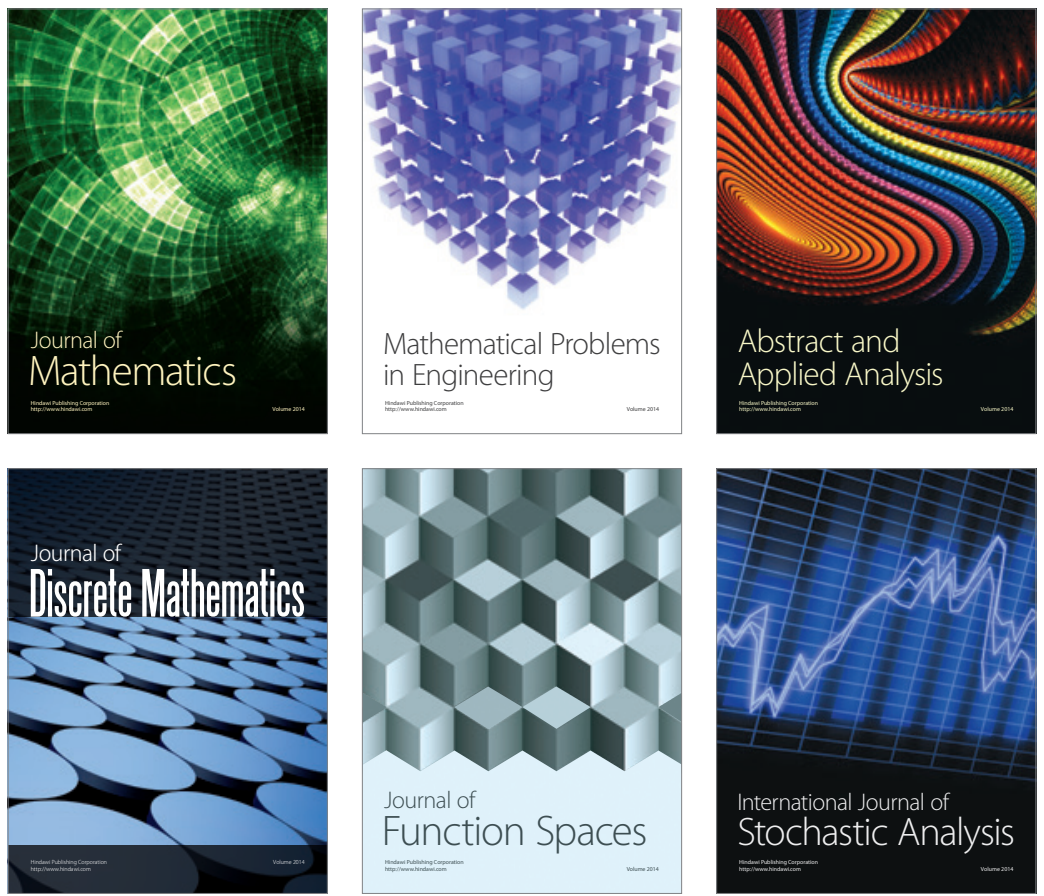

Journal of

Function Spaces

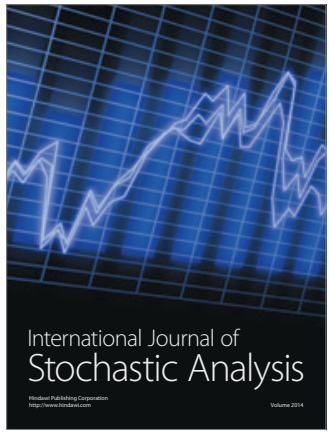

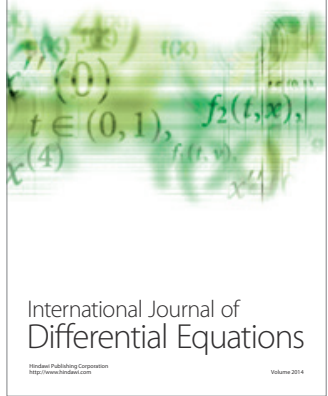
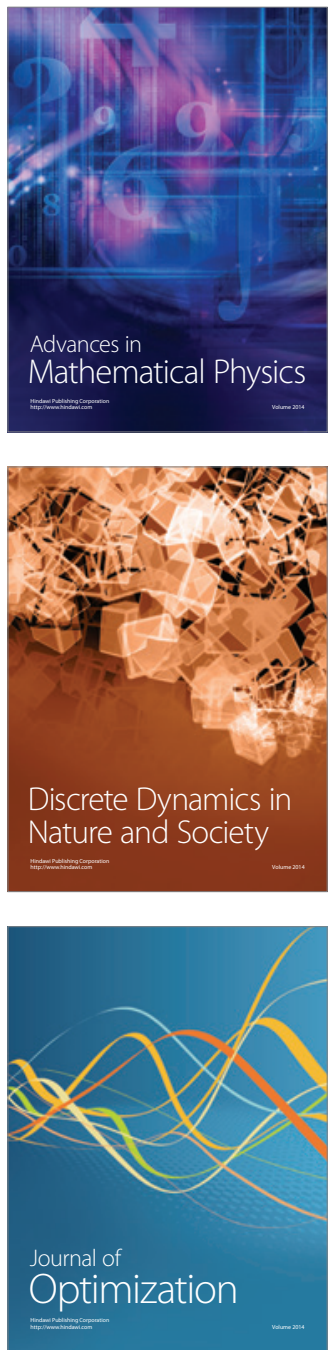